\title{
دافعية استخدام التعلم التعاوني لتنمية تعليم مهارة الكلام
}

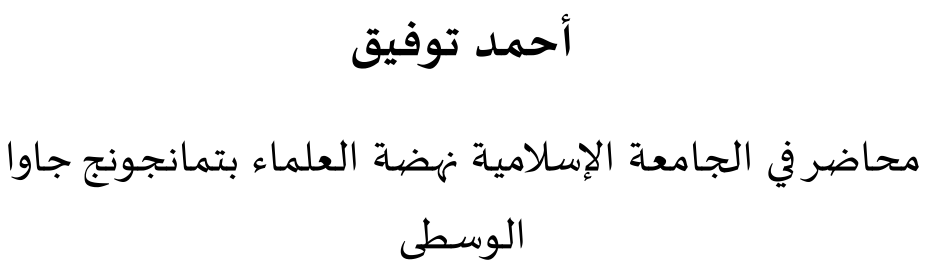

Email : ahmadtaufiq982@gmail.com

\section{Abstract}

Language is basically speech, but writing is an attempt to represent speech. It is proven that man knows speech before writing long time ago. Writing emerged in a later period of human history. The child learns to speak before he can learn to write, which he begins to learn when he enters school. All good people speak their mother tongues fluently and there are many people do not know how to write in their own languages. There are some languages still spoken in unwritten way. Cooperative learning is not where students who work together to achieve their goals unless they cooperate with each other. The mere distribution of students to groups and informing them to work together does not fall under the name of cooperative learning, even if these are the most important features and main features, there is a wide difference between the distribution of students on groups to work, and the consolidation of the concept of cooperation and rooted in the minds of students with special needs. 
أ- - أ- مقدمة

إن اللغة كما استخدمها الناس هي ما يعبر بهاكل قوم عن أغراضهه'، وقد اكد في تعريفها الشيخ مصطفى الغلاييني بأن اللغة هي الفاظ يعبر بها كل قوم عن مقاصيدهمّوقد عرفها ابن جني بأهها أصوات يعبر بها كل قوم عن أغراضهمَ لأن اللغة هي الكلام المنطوق لا الكلام المكتوب. هذه العبارة تدلنا إلى معرفة أن أساس اللغة هو الكلام. وتعد مهارة الكلام إحدى المهارات اللغوية الأسـاسية, لأن اللغة في الأصل كلام. ومهارة الكلام هو التعبير الشفوي باللغة العربية للاتصال اليومي. .فالكام يعتبر شيئا أساسيا في منهج تعليم اللغة الأجنبية. ويعتبره القائمون على هذا الميدان من أهم أهداف تعليم اللغة الأجنبية

ويشير موسى إلى فوائد التعلّم التعاوني من الناحيتين الاجتماعية والتربوية، بتوفيرها فرص التعاون بين الطلاب الذين يتعلّمون كيفيه التعبير عن أنفسهم من خلال مشاركة

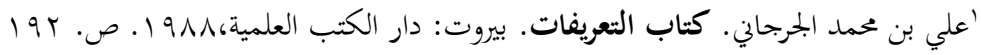

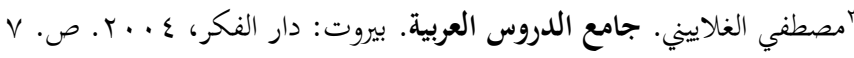
rابو الفتح عثمان بن جني. الخصائص (مصر: دار الكتاب المصري، دون السنة). ص. rr

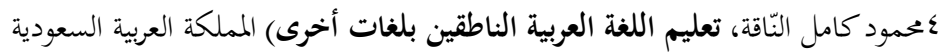


الأقران في النقاش والمحاورة،كما يكون بمقدورهم الاستيعاب بشكلٍ أفضل، وتقبل النقد من الآخرين، وهذا يكون التعلّم.

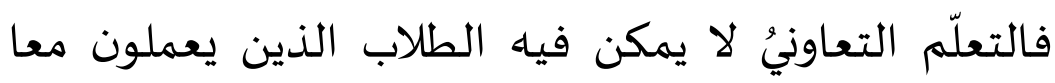

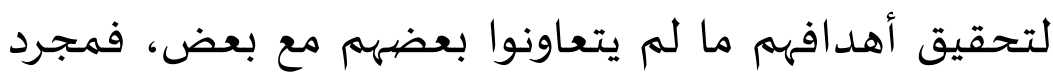
توزيع الطلاب على مجموعات وإبلاغهم أن يعملوا معا لا يندرج تحت مسمى التعلّم التعاوني، حتى ولو كانت هذه أهم وأبرز

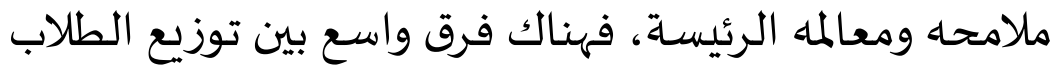
على مجموعات للعمل، وبين ترسيخ مفهوم التعاون وتأصيله في أذهان الطلاب ذوي الاحتياجات الخاصة .وأكد الموسى "أن

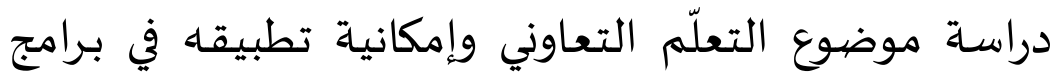

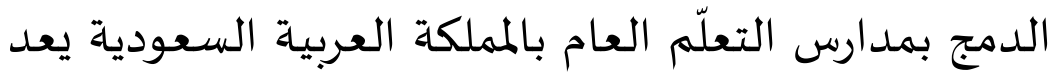

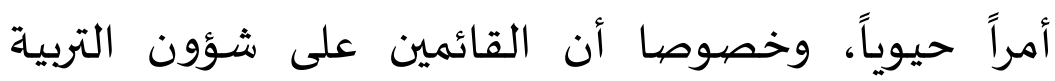
الخاصة يسعون إلى تفعيل دور المدارس العادية في مجال تربية وتعليم الطلاب غير العاديين؛ وذلك من خلال دول دمج إلى هؤلاء الطلاب في تلك المدارس". ب- بحث

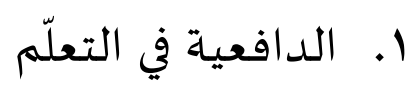
الدافعية هي تكوين فرضي يستدل عليه من سلوك الكائن الجي وبالتالي يستخدم مفهوم الدافعية لتحديد اتجاه 
السلوك وعليه فإن كل واحد منا يكون على وعي بمختلف دوافعه. ْورأي على عسكر أن الدافعية هي الرغبة في القيام بعمل يحقق الفرد من ورائه حاجة أو هدفا مرغوبا.? الدافعية هي قوة داخلية تحرّك الفرد لأن يفعل ما من الأنشطة لتحقيق الأهداف. وهي في عملية التعليم والتعلم تدعي إلى إتمام العملية لأن كلما كان وراء المتعلم دافع يستحثثه وحافر يشده إلى تعلم شيئ ما، كان ذلك أدعى إلى إتماماه وتحقيق الهدف مناه خاصية في الأشكال المعقدة ومهاراته المتشابكة.

أما بالنسبة إلى دافعية تعلم الطلبة اللغة العربياة، تنقسم الدافعية لتعلم اللغة الأجنبية إلى قسمين، وهي فيما :

www.pdffactory.com،نسرين عدنان عثمان، دون سنة، الدافعية نحو التعليم"

جعلى عسكر، الأسس النفسية والاجتماعية للسلوك في مجال العمل

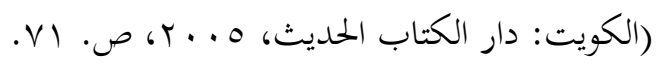

لحمد كامل الناقة ورشدى أحمد طعيمة، طريقة تلدريس اللغة العربية لغير الناطقين بها

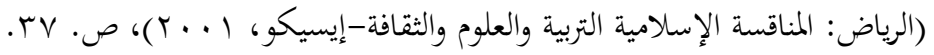

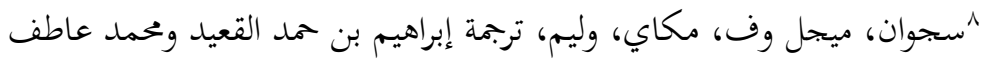

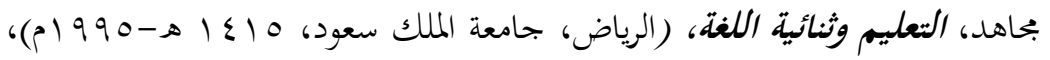
$.9 \Lambda-9 \vee \cdot ص$ 
أولا : الدوافع النفعية المتعلم يهدف إلى إدراك المزايا الكامنة في معرفة لغة ما والتمكن منها، وتتراوح هذه المزايا من الحاجة إنها

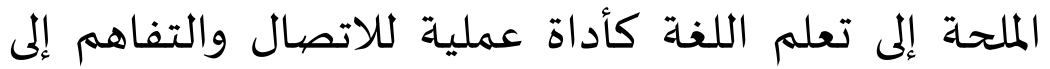
التمكن منها لضمان مستقبل وظيفي أفضل.

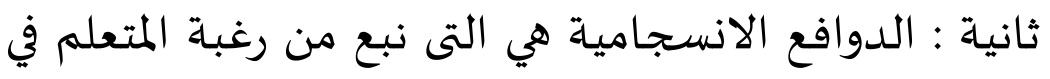

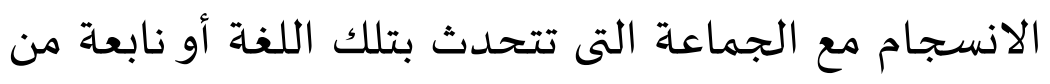

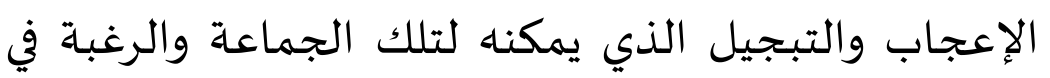
إظهار الاقتران بها ولو من ناحية مثالية على الأقل.

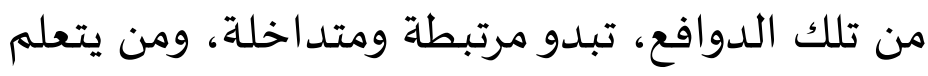
اللغة بقصد الاندماج في الجماعة يؤمن بأنه سيجلب في تبداه الجماعة الجديدة منافع تساعده على الحياة في المجتمع.

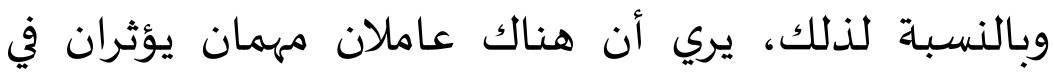

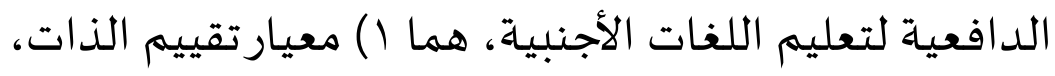

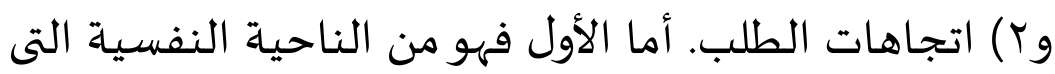

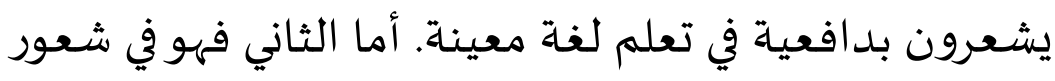
الطالب بخفة الأنشطة المختلفة في تعليم اللغات الأجنبية

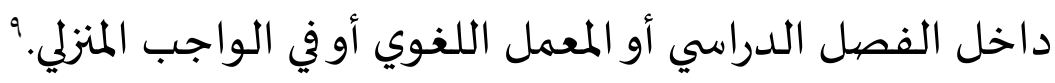

9 سوتامان، دافعية تعلم اللغة العربية لدي تلاميذ المدارس الابتدائية (دراسة

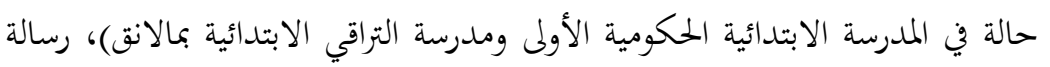

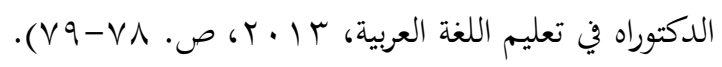


ولذلك، تعليم اللغة يجب أن يرتبط بطموح الطلاب حتي كانت اتجاهات الطالب وما يرتبط بدافعيته من الأمور الضرورية في تعلم اللغة الأجنبة. وهذا الاتجاه دوره مهه حتي يدل عليه ما تقوم باه بعض الجامعات في الوقت الحاضر من لعن

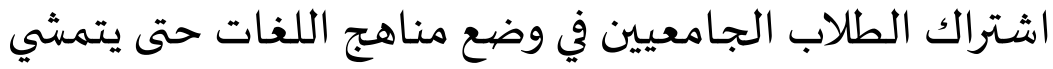
ذلك مع اتجاهاتهم وعلى أساس دافعيتهم في التعلم. ' في عملية التعليم والتعلم، يحتاج الطلاب إلى تنمية الدوافع في التعلم لأنها تفيد في ترك الخجل والقلق والوسوسة في إجراءها. تظهر الثقة بالنفس من تحضير الدافعية في الفصل مع تنشيط الحركات المساعدة. وكانت الدافعية يمكن حضورها من الأمور الداخيلية والخارجية، من الأمور الداخلية التى تؤدي إلى وجود الدافعية هي ميول الطلاب في مئوني

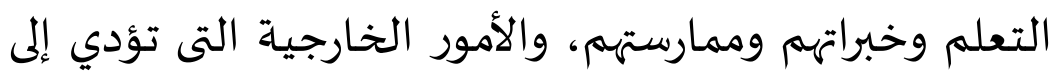

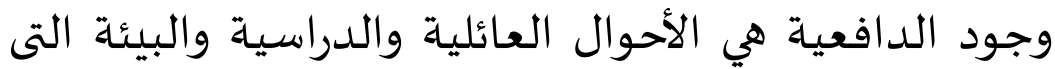
يعيشون فيها في حياتهم اليومياة. لذلك، طور ماسلو (Maslow)

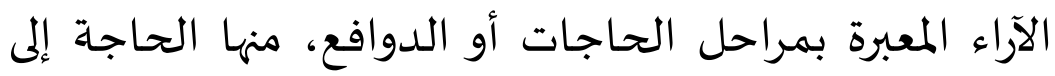

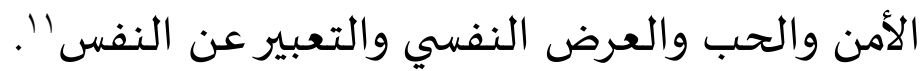

·'منصور، عبد المحيد سعد أحمد، علم النفس اللغوي (الرياض، جامعة الملك

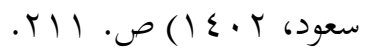

${ }^{11}$ Martinis Yamin, Paradigma Baru Pembelajaran (Jakarta: Gaung Persada Press, 2011), hlm. 217 
في عملية التعليم والتعلم، يحتاج الطلاب إلى تنمية

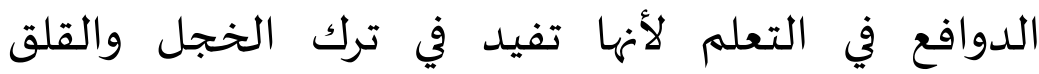
والوسوسة في إجراءها. تظهر الثقة بالنفس من تحضير الدافعية في الفصل مع تنشيط الحركات المساعدة. وكانت الدافعية يمكن حضورها من الأمور الداخيلية والخارجياة، من الأمور الداخلية التى تؤدي إلى وجود الدافعية هي ميول الهور الداحيه

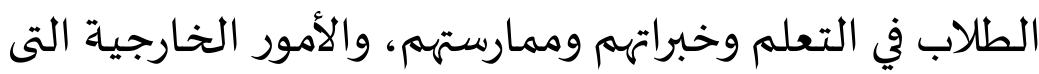
تؤدي إلى وجود الدافعية هي الأحوال العائلية والدراسية

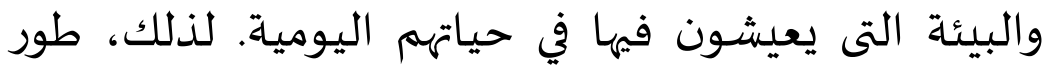

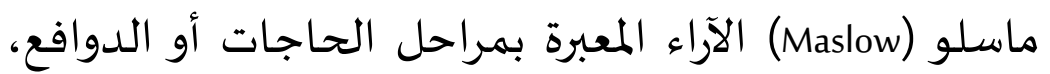

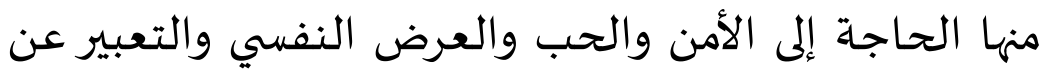

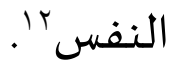

في عملية التعليم والتعلم، يحتاج الطلاب إلى الدوافع، ألمعاج فإذا أهملها المعلمون فسيجري التعليم والتعلم دون فعالية جيدة. وتوجد الدوافع حينما تتوافر الأمور التى يحتاجون

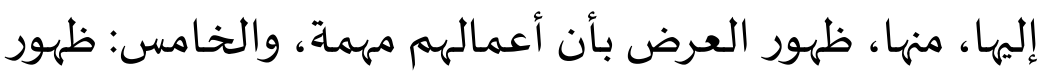

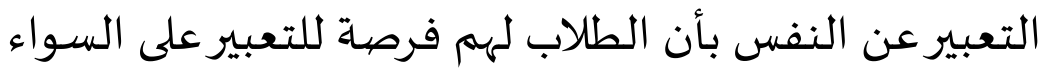
عن النفس دون اختلاف بينهم والآخرون وهلم جرى. 
وإضافة إلى ما سبق، لابد للمعلم أن يبنى الدوافع في التعلم الصفي بشكل ما يلى:

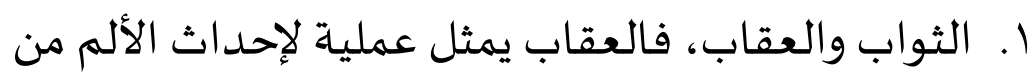
أجل أن يتخلى الطلاب عن الاستجوابات الخاطئة ويبني

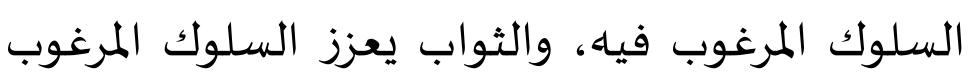
فياه.

r. المنافسـة، تستعمل المنافسة على نطاق واسع داخل وخارج الفصل الدراسي فهي تمثل حافزا قويا وحيويا في مجال التعلم وتؤدي بالضرورة إلى تحسين أداء الأطفال

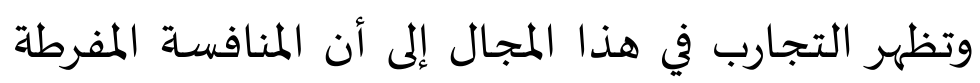

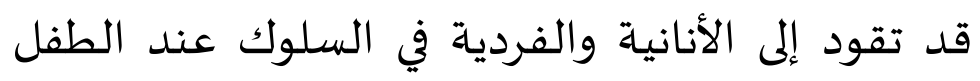

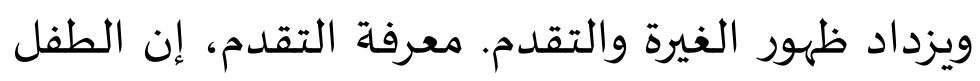
الذي يقف على التقدم الذي يحرزه في معرفة ما يحصل فيل ولئل على تشجيع ودافة أكثر من هذه المعرفة وتشير التجارب التحري

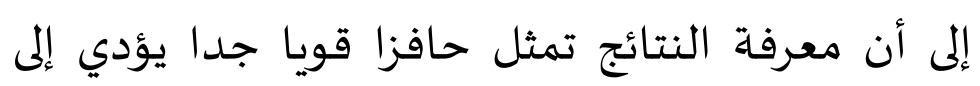
تحسين أداء الطلاب في عملية التعليم والتعلم.

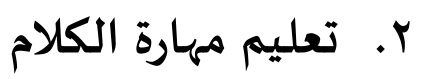
أ. مفهوم تعليم مهارة الكلام () تعريف التعليم 
تعليم لغة مصددر من علّم - يعلم- تعليما أي جعله

يعلم. واما التعليم اصطلاحا هناك تعاريف كثيرة، منها:

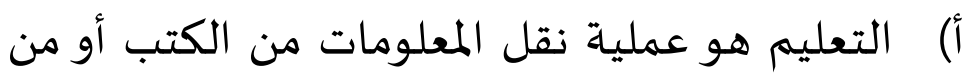

عقل المعلم إلى عقل المتعلم

ب) التعليم هو نقل المعلومات من المعلم الإيجابي إلى المعلى

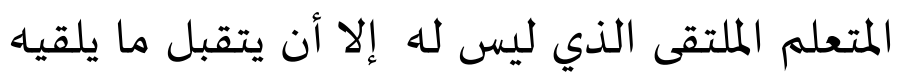

$$
\text { المعلمء ا. }
$$

ج) التعليم بشكل عام هو أنه عملية إعادة بناء الخبرة

التى يكتسب المتعلم بواسطتها المعرفة والمهارات

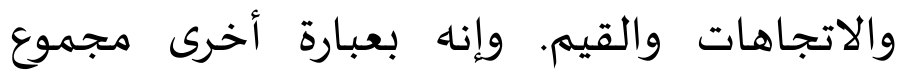
الأساليب التى يتم بواسطتها تنظيم عناصر البيئة

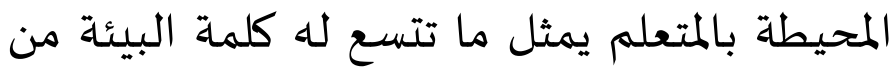

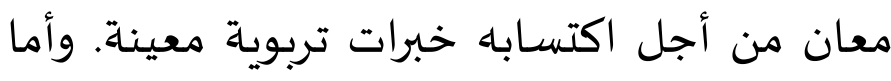
مفهوم التعليم بشكل خاص هو نشاط مقصود يقوم

"امسن شحاته، تعليم اللغة العربية بين النظرية و التطبيق، (مصر : المكتبة المصرية البنائية،

$$
19.0) \text { (Y..人 }
$$

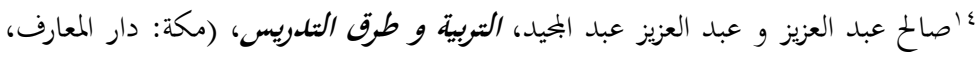

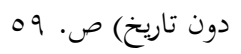


باه فرد آخر على اللاتصال بنظام من الرموز اللغوية يختلف عن ذلك الذى ألفه وتعود الاتصال بهاء 1. د) التعليم هو إيصال المعلم العلم والمعرفة إلى أذهان التلاميذ بطريقة قويماة، وهي الطريقة الإقتصادياة التي توفر لكل من المعلم والمتعلم والوقت والجهد في سبيل الحصول على العلم والمعرفة 17

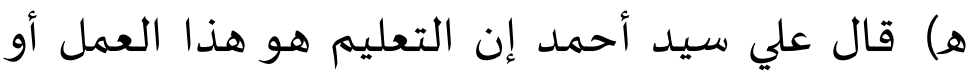
العلم الذي يطبق في صورة كتب ومناهج وأنشطة،

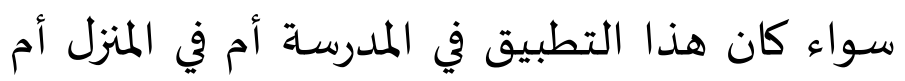

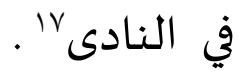
و) التعلم هو عملية تعديل في السلوك أو الخبرة؛'. من التعريفات السابقة يلخص الباحث أن التعليم

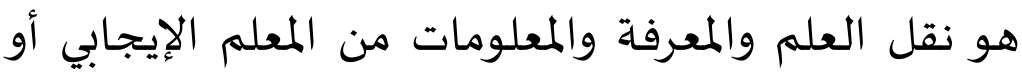

•ارشدى أحمد طعيمة، تعليم اللغة العربية لغير الناطقين بها مناهجه و أساليبه، ( مصر:

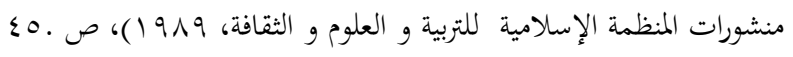

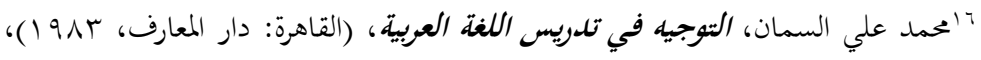
ص. IT طا'علي سيد أحمد، التعليم والمعلمون، (مكة المكرمة: دار الصابوني ودار ابن حزم، دون سنة)، ^'فهمي مصطفي ، فسكولوجية التعليم، (مصر : مكتبة مصرية، د.ت)، ص بr ب 
الكتب إلى أذهان المتعلم الملتقى بطريقة قويمة ومناسبة لتحقيق الأهداف المنشودة.

الكلام في أصل اللغة عبارة عن الأصوات المفيدة،

وعند المتكلمين هو المعنى القائم بالنفس الذي يعبر عنده

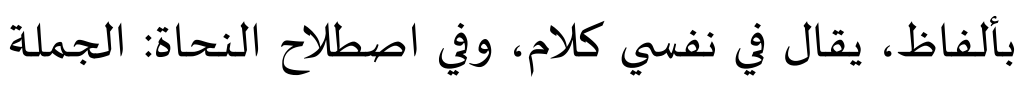

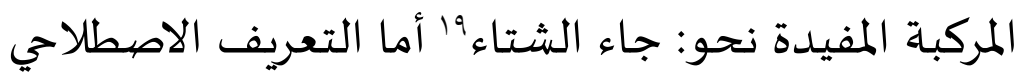

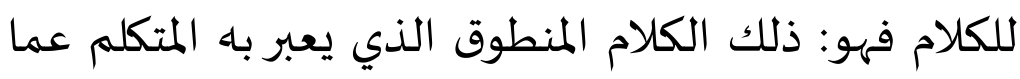

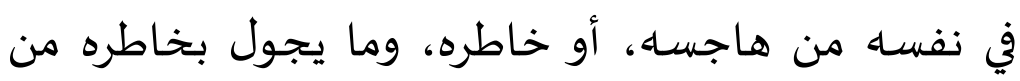

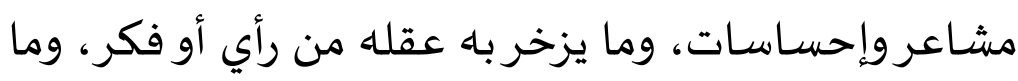

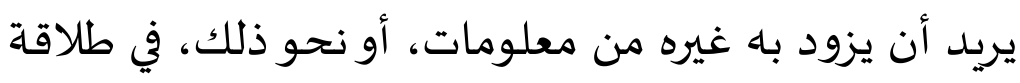

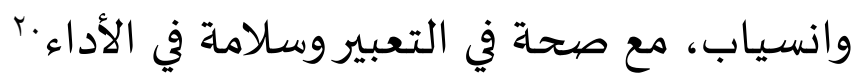
r

المهارة هي مصيدر من مهر - يمهر- مهارة ـ المراد فيها

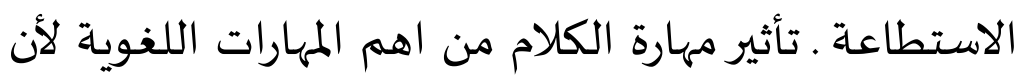

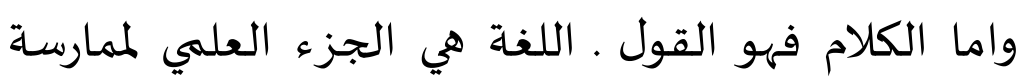

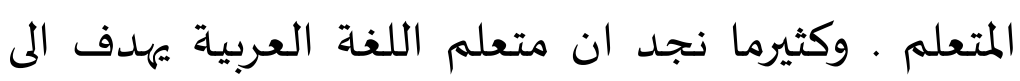

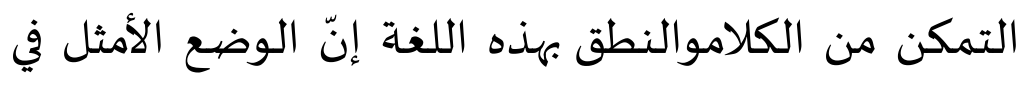

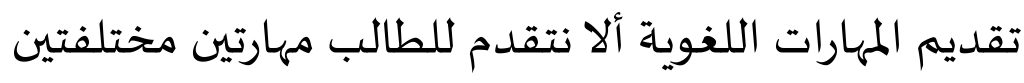

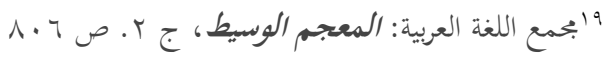

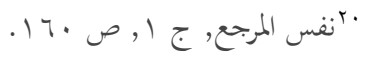

Jurnal Izdihār : Journal of Arabic Language Teaching, Linguistics, and Literature 
في وقت واحد ، كأن ندربه على تركيب جمل جديدة من

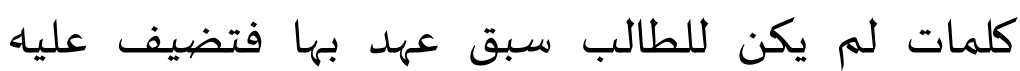
صعوبتين : إحداهما ضرورة فهم الكلمات الجديدة والأخرى يهري تركيب جمل جديدة. ومن الملاحظ في بعض كتب تعليم العربية أنّ تدربباتها تشتمل على جديده ومن مفردات وتراكيب لم ترد

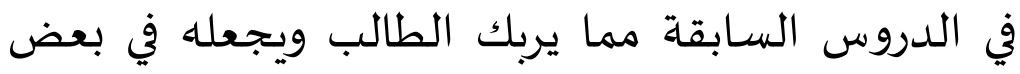

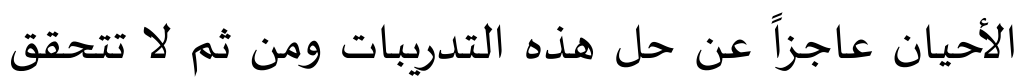
أهد افها

عرفنا ان لمهارة اللغوية تتكون من اريع مهارات , مهارة الكلام ومهارة الاستماع ومهارة الكتابة ومهارة القراءة .

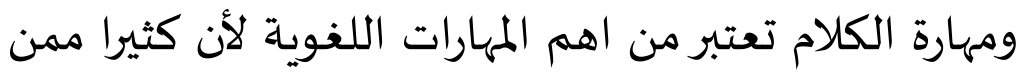

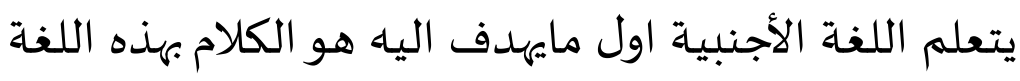

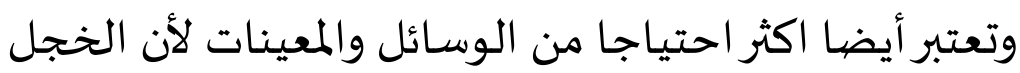

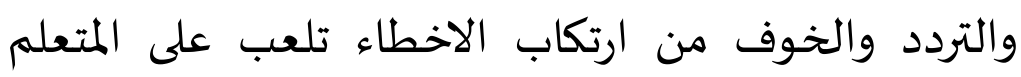

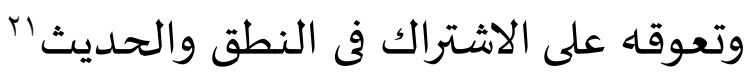
r) (r) (بريف الكلام

يقول اللغويون إن الكلام هو اللغة. فالكلام مهارة

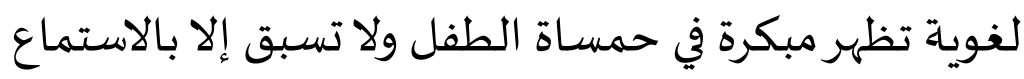

21 Henry Guntur Tarigan, Berbicara Sebagai Suatu Keterampilan Berbahasa (Bandung : Angkasa, 1990) hal 1 
فقط، ذلك الذي من خلاله يعلم الكلام. ولا شك أن الكلام

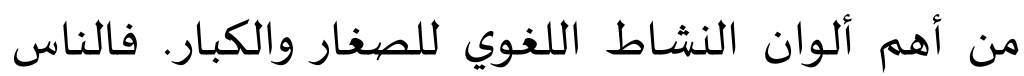
يستخدمون الكلام أكثر من الكتابة، أي أنهم يتكلمون أكثر لهن

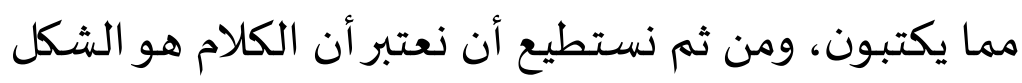

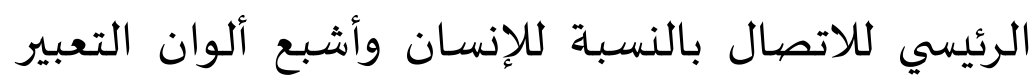
وأكثرها قدرة على ترجمة المشاعر بشكل مباشر كما أنه أيضا

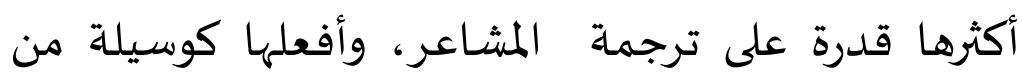

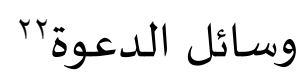

والكلام في اللغة الثانية من المهارات الأسساسية التي تمثل غاية من غايات الدراسة اللغوية. وإن كان هو نفساء

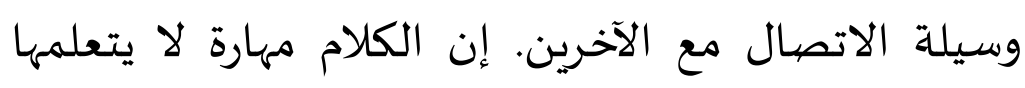

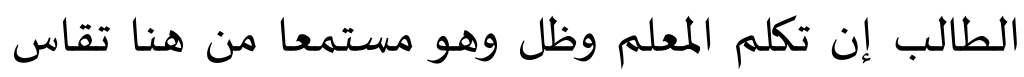

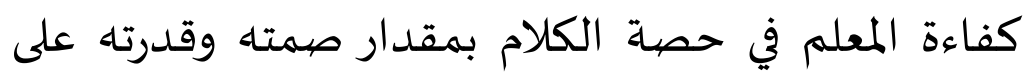
توجياء الحديث وليس بكثرة كلامهاه واستثاره الحديث. ومن بهن

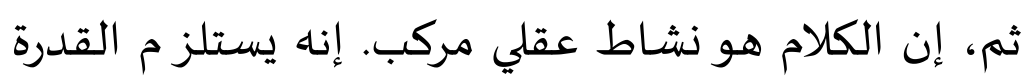

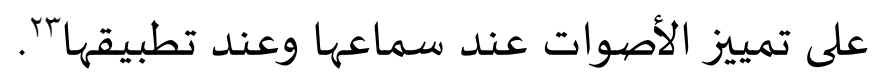

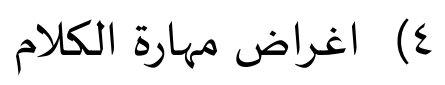

\footnotetext{
rاrممود كامل الناقة وآخرون، تعليم اللغة العربية أسسه وإجراءته (مصر: دون الناشر،

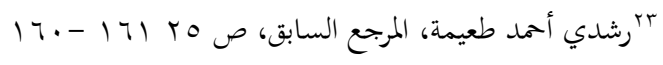




$$
\begin{aligned}
& \text { أن ينطق المتعلم أصوات اللغة العربية } \\
& \text { أن ينطق الأصهوات المتجاوزة و المتشابهة }
\end{aligned}
$$

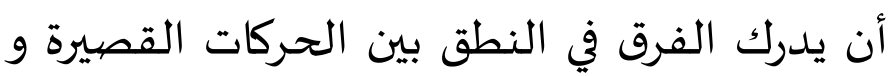

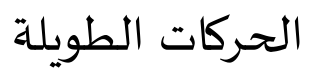

أن يعبر عن أفكاره مستحدما الصيغ النحوية

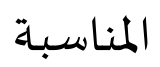

أن يعبر عن أفكاره مستحدما النظام الصحيح

$$
\text { لتركيب الكلمة في العربية خاصة في لغة الكالام }
$$

أن يستخدم بعض خصائص اللغة في التعبير الشفوي مثل التذكير و التأنيث و التمييز العداد و الحال و نظام الفعل و أزمنته و غير ذالك مما يلزم

$$
\text { المتكلم بالعربية }
$$

أن يكتسب ثروة فظية كلامية مناسبة لعمره و مستوى نضجاه و قدارته، و أن يستخدم هذه الثروة

$$
\text { في إتمام عمليات اتصال عصرية }
$$

أن يستخدم بعض أشكال الثقافة العبية المقبولة و المناسبة لعمره و مستواه الاجتماعي و طبيعاة عمله، و أن يكتسب بعض المعلومات الأسساسية عن التراث

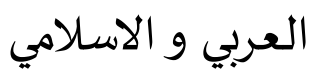


أن يعبر عن نفساه تعبيرا واضحا و مفهوما في مواقف الحديث البسيطة أن يتمكن من التفكير باللغة

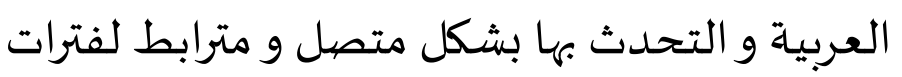

$$
\text { زمنية مقبولة؛؟r. }
$$

\section{ع. أهداف تعليم مهارة الحديث ( الكلام ) :}

أن ينطق المتعلم أصوات اللغة العربية ، وأن

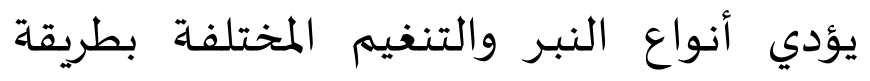

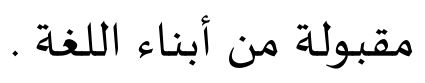

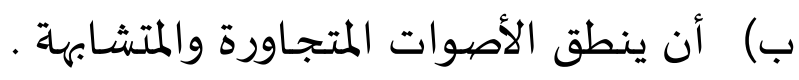

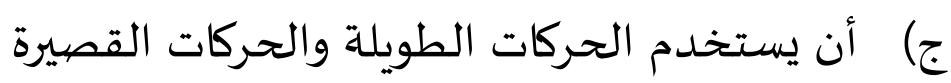

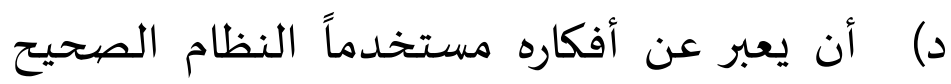

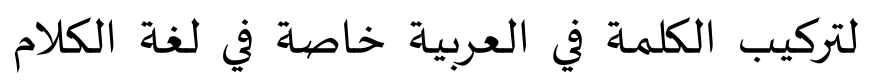

الفصحى . أن الفيب

ه) أن يكتسب ثروة لفظية كلامية مناسبة لعمره

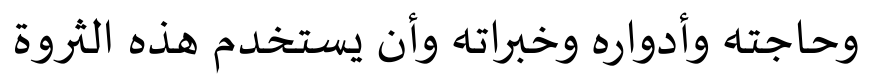

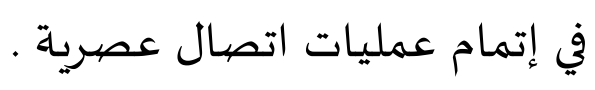

\footnotetext{
ع بحمدد كامل الناقة، علي بن محمد الجرجاني. كتاب التعريفات. بيروت: دار

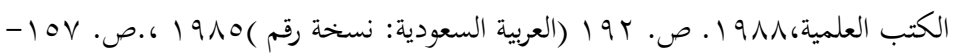


و) أن يستخدم بعض أشكال الثقافة العربية الإسلامية المناسبة لعمره ومستواه لئاه الثقافي وطبيعة عمله ، وأن يكتسب بعض المعلومات الأساسية عن التراث العربي الإسلامي .

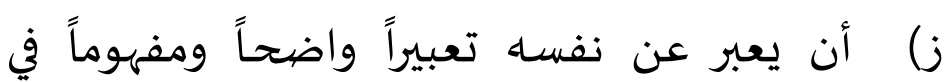
مواقف الحديث البسيطة . ح) أن يفكر باللغة العربية ويتحدث بها بشكل متصل البهل ومترابط لفترات زمنية مقبولة .

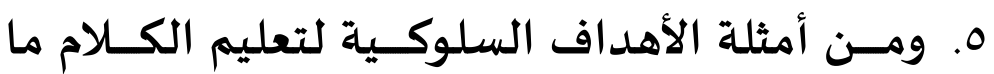
يلي: أن يطلب المتعلم شيئًاً ما . أن يتعلم عن الأماكن والأوقات والأشخاص . أن يطلب من الآخرين عمل شيء ما . أ. أن يقيم علاقات أليفة مع أبناء اللغة . . أن يحكي قصة بسيطة ، أو يقول شيئاً للآخرين .

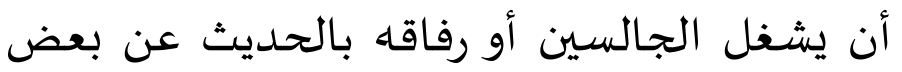

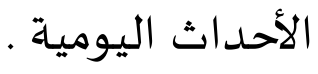$$
\text { أن يفهم الآخرين ويوجههم ويرشدهم . }
$$ 
أن يقضي حوائجـه اليومية ويؤدي ما يطلب منه من

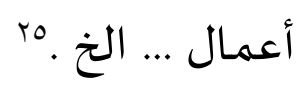

$$
\text { أ. ألتعلم التعاوني التعاوني }
$$

هو طريقة حديثة في التعليم تبتعد في أسلوبها

عن الطرق التقليدية، كاللوح والطبشورة، حيث إنّها

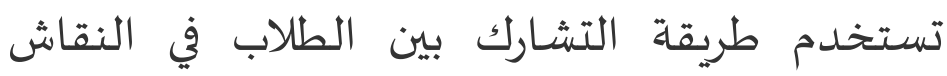
يجلسون بشكلٍ دائري ويعرض كل طالب أفكاره ووجهة

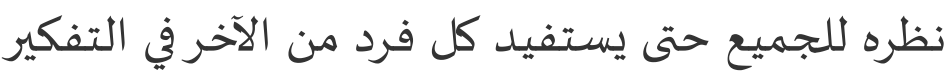

$$
\text { والوصول للحلول }
$$

تنظيم زمري من خلال تقسيم الطلاب إلى

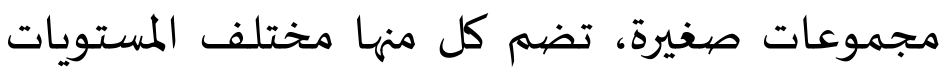

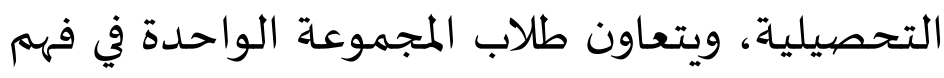

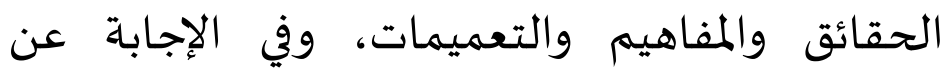

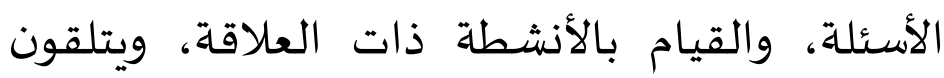

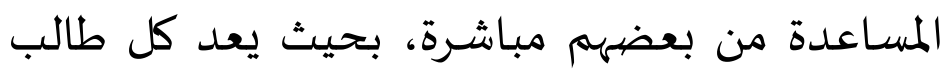

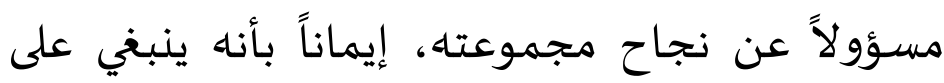

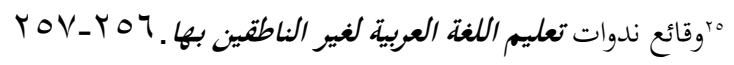


المجموعة أن تعمل على تحقيق الهدف ويكون دور المعلم تقديم مذكرات مصاغة على شكل أهداف وأساليب وأنشطة وتقويم، وإعطاء تعليمات تخبر

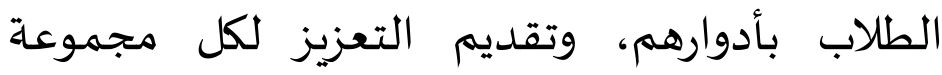

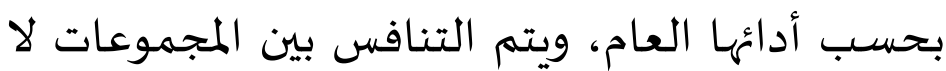

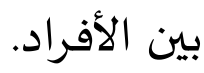
ب) بوائد التعليم التعاوني هذه الطريقة من التعليم تخفف على الطلاب الرهبة من الدراسة والخوف من طريقة التعليم التقليدية التي ينفرد بها المعلم في التفكير. تبادل الأفكار

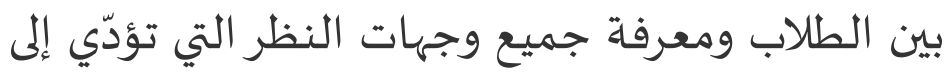
اكتشاف حلول ونتائج جديدة ومهمة

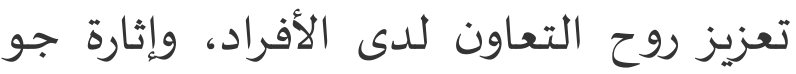

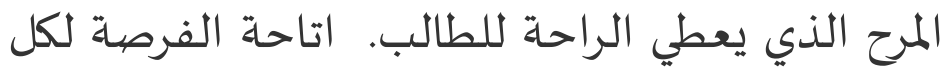
طالب بأن يسأل زملاءه ويستعين بهم بدلاً من المعلم عندما يحتاج لذلك. طريقة التعليم التعاوني تستخدم

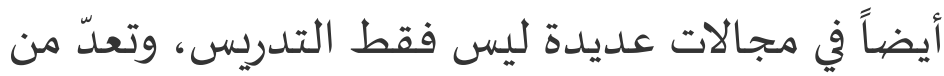
أهم الطرق في العلاج النفسي الجماعي فيستمع المرضى مئى لبعضهم البعض ويطرح كل مريض حل لمشكلة الآخر. 


\section{ج) أنروط التعليم التعاوني}

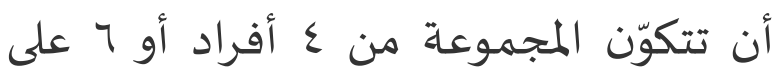

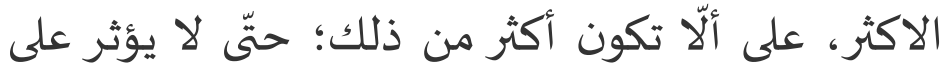

تركيز المجموعاة ويشتّتهم.

() لا يعتمد كل من الطلبة على الآخر فهذه الطريقة

تقوم على التعاون فيما بينهم والعمل الجماعي.

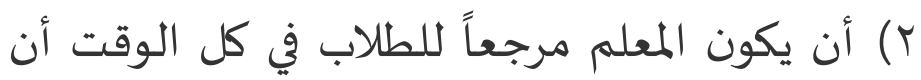

$$
\text { يسألوه بما لا يعرفوه. }
$$

r) أن يتناقش الطلاب مع بعضهم البعض وأن يأخذ لون

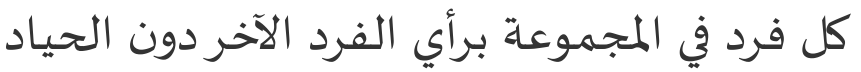

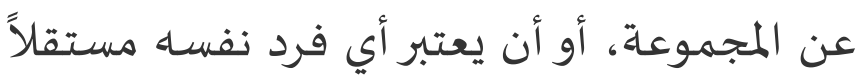

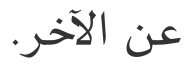

ع) الانضباط وعدم استغلال الوقت المعطى

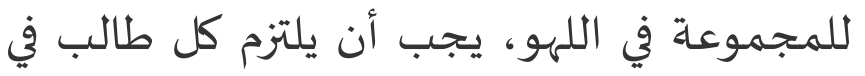

المناقشاة وعدم التكلم بلا فائدة.

\section{د) طريقة التعليم التعاوني}

أن يحدّد المعلم أفراد المجموعة التي تتكوّن من 7

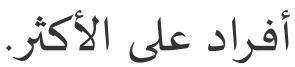

يعطي لكل فرد من أفراد المجموعة رقماً يعرّف باء. 
تسمّى كل مجموعة باسمٍ محدد أو تعطى لقباً لمعرفتها.

يعطي لكل مجموعة موضوعاً ليبحثوا ويتناقشوا

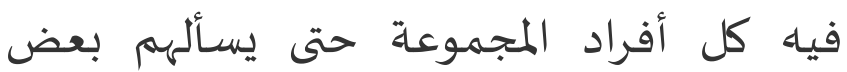
الأسئلة، بعد أن يتنتهوا من النقاش وطرح الأفكار

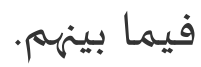

يحدّد المعلم فرداً في المجموعة يمثلها ويتكلم بالنيابة عنها، ولكن في بعض الأحيان يفضل فردان

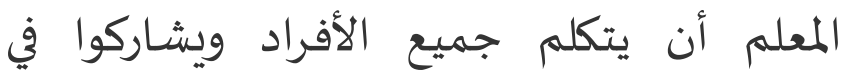
الحديث والنقاش للتأكد من فهم جميع الأفراد للموضيوع.

بعد الانتهاء من النقاش مع جميع أفراد المجموعة

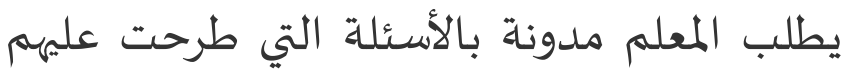
مع إجاباتها. يجب أن يحدّد المعلم مدى نجاحه من تحقيق

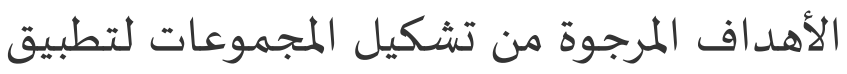
طريقة التعليم التعاوني. تعدّ طريقة التعليم التعاوني من أنجح الطرق على

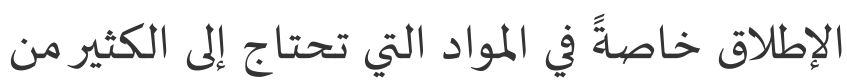


المناقشة والتواصل بين المعلم والطلبة، فهذه الطريقة سهّلت النقاش فيما بينهم دون إثارة

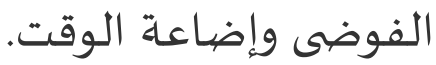
ه) خصيائص التعلم التعاوني من التعريف السابق يمكن لنا أن نشتق عددا من التعاوي

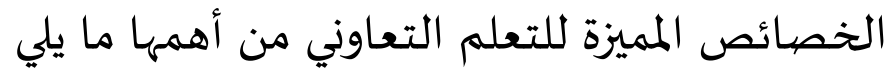
التعلم التعاوني صيغة متعددة الاستراتيجيات للتدريس تقوم على تنظيم الفصل الدراسي في صورة مجموعات

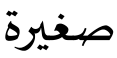

التفاعل بين الطلاب داخل المجموعات خاصية مميزة للتعلم التعاوني تجعل مناه صيغة تعليمية مميزة تساعد على إنجاز الأهداف في مستوى الإتقان المطلوب. يتسم التعلم التعاوني بالاجتماعية في أداء أدوار التعلم، حيث يتم التعلم في سياق احتكاك اجتماعي

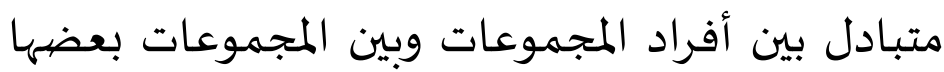
البعض، وبينهم وبين المعلم

يعتمد التعلم التعاوني على جهدي المتعلم والمعلم معا، ولمبن

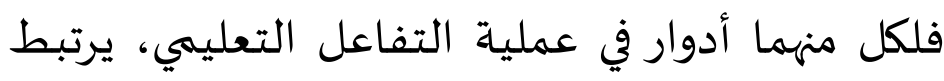

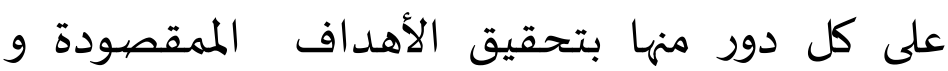


التعلم

من

المنشودة

و التعاون وتقديم المعونة والمساعدة بين أفراد

المجموعات سمة مميزة لهذا النوع من التعليم، تجعل مناه صيغة من الصيخ الفريدة التي تعمل على تكامل خبرات المتعلمين. الشـروط الواجب توافرها في التعّلم التعاوني:

عند إتباع أسلوب التعّلم التعاوني يلزم إتباع الشـروط التالية: () أن يتوافر الاعتماد الإيجابي المتبادل بين أعضياء الجماعـة. r) إتاحة الفرصية للتفاعل المباشر بين أفراد الجماعـة. r) الاستفادة القصيوى من إمكانيات ومهارات كل عضيو من أعضياء الجماعة. ع) أن يكون كل عضيو في الجماعة مسؤولاً يعهد إليه من أعمال. 0) أن تتحقق الأهداف ومهارات العمل الجماعي بين أعضياء

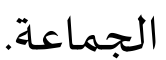
7) ألا يزيد حجم الجماعـة في التعّلم التعاوني عن ستاة طلاب. V يفضل أن تكون الجماعة من مختلف القدرات غير المتججانسة. ^) أن يكون جنس الجماعة من فئة واحدة. 
9) تحديد فنيات التعّلم التعاوني التي يتم استخدامها وفقاً لطبيعة الموقف التعليمي.

• (1) أن يكون للتلميذ إيجابية في العمل مع زملائه داخل

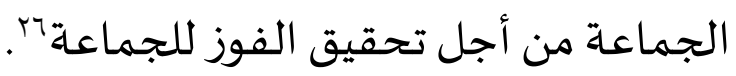

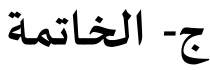

1. فوائد التعّلم التعاوني من الناحيتين الاجتماعية-والتربوية، التهاهِ بتوفيرها فرص التعاون بين الطلاب الذين يتعَّمون كيفياء

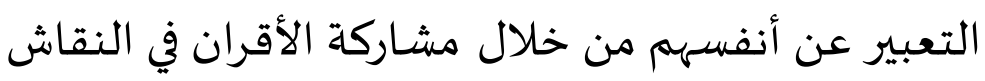

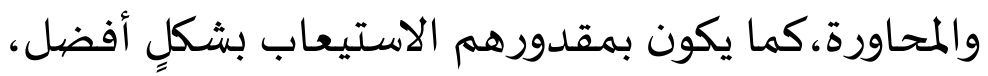
وتقبل النقد من الآخرين، وبهذا يكون التعّلم فعالاً.

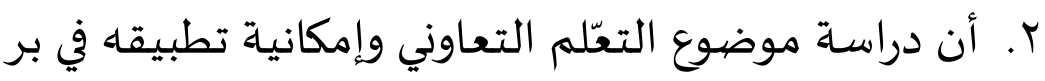

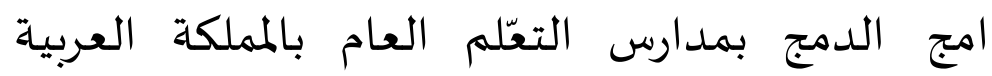

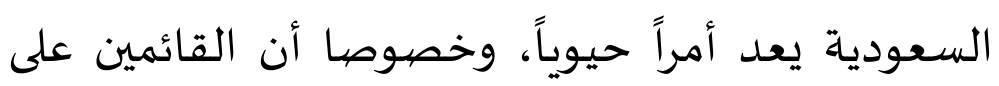

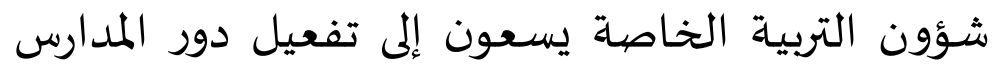

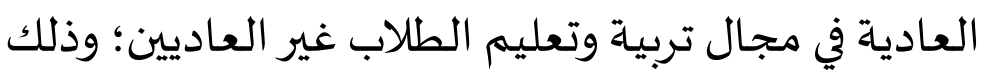

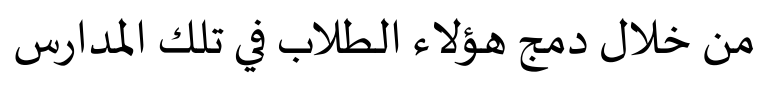

$$
\begin{aligned}
& \text { بrالديب، محمد مصطفى م" علم النغس التعلم }
\end{aligned}
$$

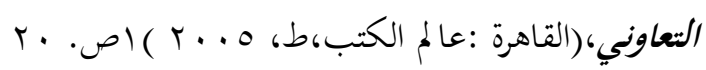


r. وضع الأطفال ذوي الاحتياجات الخاصة في المدارس العادية

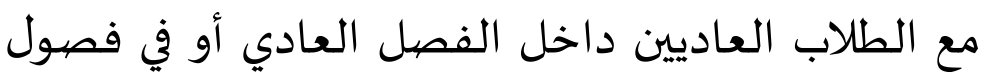
خاصة ملحقة لبعض الوقت، أوطوال الوقت حسب ما

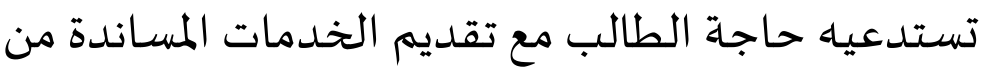
خلال فريق متعدد التخصصيات وإجراء التعديلات الضرورية المادية والبشرية لتسهيل فرص نجاحهم وتقدمهم.

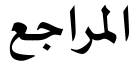

الجرجاني علي بن محمد. (1911) كتاب التعريفات. بيروت:

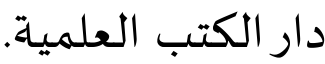

الغلاييني مصطفي.(ع . . ب). جامع الدروس العسبية. بيروت:

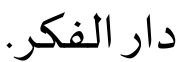
جني ابو الفتح عثمان بن. الخصبائص ، مصر: دار الكتاب المصري، دون السنة.

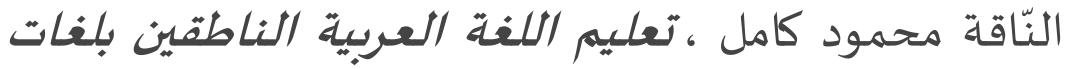

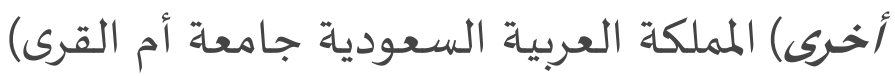

عثمان نسرين عدنان ، دون سناة، الدافعية نحو التعليم، www.pdffactory.com عسكر على ( . . . ) ، الأسس النفسية والاجتماعية للسلوك في مجال العمل الكويت: دار الكتاب الحديث. 
طعيمة محمد كامل الناقة ورشدى أحمد (1 ..ب)، طريقة تلدرليس اللغة العربية لغير الناطقين بها الهيه الرياض:

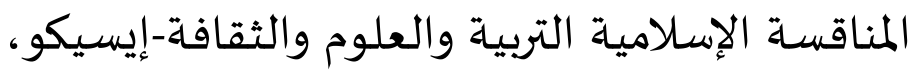
سجوان، ميجل وف، مكاي، وليم ( 10 (أه)، ترجمة إبراهيم بن حمد القعيد ومحممد عاطف مجاهد، التعليم وثنائية اللغة، الرياض، جامعة الملك سعود،

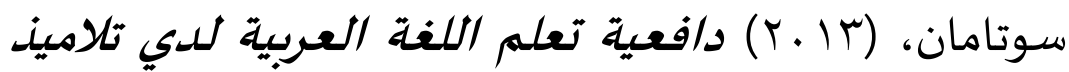
المدارس الابتدائية دراسة حالة في المدرسة الابتدائية

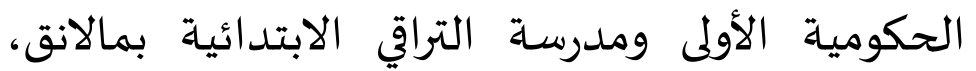
رسالة الدكتوراه في تعليم اللغة العربية.

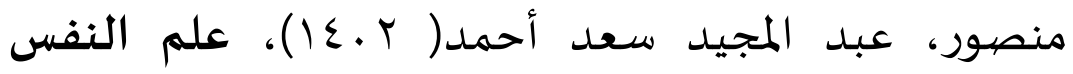
اللغوي ، الرياض، جامعة الملك سعود.

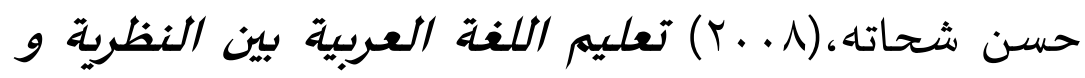
التطبيق، مصر : المكتبة المصرية البنائية. المجيد صالح عبد العزيز و عبد العزيز عبد ، التربية و طرق المقبه

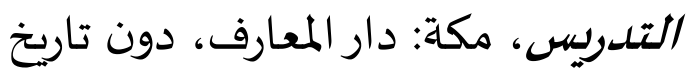

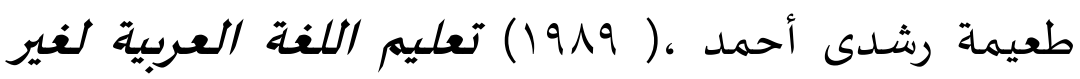

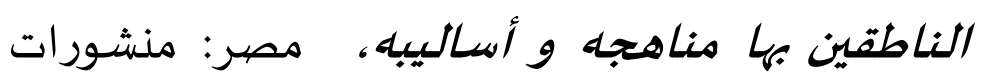
المنظمة الإسلامية للتربية و العلوم و الثقافة. 


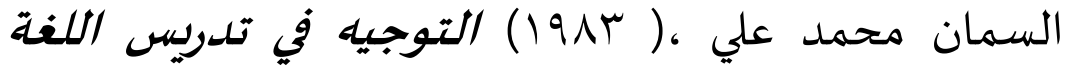
العربية ، القاهرة: دار المعارف.

أحمد علي سيد ، التعليم والمعلمون، مكة المكرمة: دار الصيابوني ودار ابن حزم، دون سنة.

مصطفي فهي ، فسكولوجية التعليم، مصر: مكتبة مصرية، دون سئه

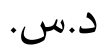

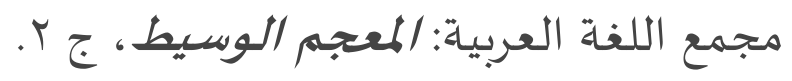

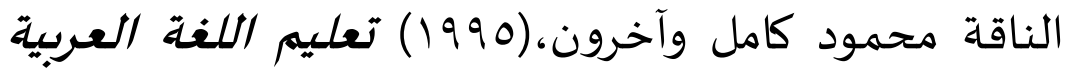
أسسسه وإجراءته مصر: دون الناشر.

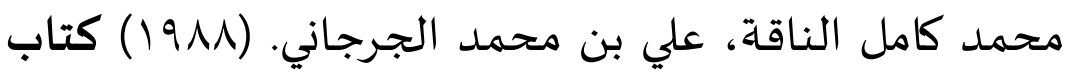
التعريفات. بيروت: دار الكتب العلمية.

وقائع ندواتة (1910 )، تعليم اللغة العربية لغير الناطقين بها. العربية السعودياة: نسخة الديب، محمد مصطفى ( T . T ) م" علم النفس التعلم التعاوني، القاهرة :عالم الكتب، مجمئ Henry Guntur Tarigan.1990, Berbicara Sebagai Suatu Keterampilan Berbahasa Bandung : Angkasa,

Martinis Yamin 2011, Paradigma Baru Pembelajaran Jakarta: Gaung Persada Press 